\title{
Vielfalt der Familienformen - mit welchen Folgen?
}

\section{Jean Martin}

Dr. med., Mitglied der Redaktion

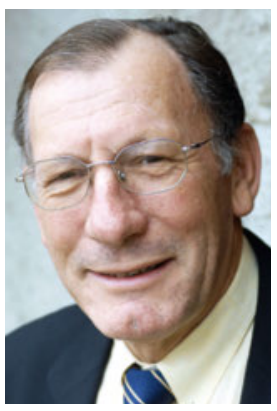

Literatur

1 Nationale Ethikkommission im Bereich Humanmedizin (NEK-CNE). Die medizinisch unterstützte Fortpflanzung. Ethische Überlegungen und Vorschläge für die Zukunft. Bern: Stellungnahme Nr. 22/2013. Vor allem im Rahmen der «European Study of Assisted Reproduction Families» (ARF) und in der «UK Longitudinal Study on ARF" Golombok, S (2015) Modern Families: Parents and Children in New Family Forms. Cambridge: Cambridge University Press.

jean.martin[at]saez.ch
Edwards und Steptoe ermöglichten 1978 die Geburt des ersten "Retortenbabys» in England. Im vergangenen September hatte die Schweizerische Gesellschaft für Psychologie in Lausanne Prof. Susan Golombok vom Centre for Family Research der Universität Cambridge eingeladen, um über «Modern families: parents and children in new family forms» zu referieren.

Bei der Geburt von Louise Brown sprach das Time-Magazin von der "most awaited birth in perhaps 2000 years" ... Dieser Erfolg hatte zwar überrascht, doch kaum jemand hatte sich vorgestellt, zu welchen Entwicklungen dies noch führen würde. Damals stellten sich zahlreiche Fragen: Wie würde sich die psychosoziale und soziologische Zukunft der aus künstlicher Befruchtung geborenen Kinder und ihrer Eltern gestalten? Wie sollten sich die praktischen und rechtlichen Fragen in Verbindung mit dergestalt auf den Kopf gestellten Begriffen - Fortpflanzung, Abstammung - lösen lassen? Im Vereinigten Königreich hatten damals höchste Rechtsinstanzen bestimmte Modalitäten untersagt, mit dem Verweis auf deren Negativwirkung und lesbischen Müttern, die (aus heterosexuellen Beziehungen) Kinder hatten, wurde das Sorgerecht entzogen! Heute werden diese Fragen überall diskutiert (bis dato wurden 6,5 Millionen Kinder mit Hilfe von IVF geboren). In der Schweiz hat die nationale Ethikkommission im Jahr 2013 einen Bericht zur medizinisch unterstützten Fortpflanzung veröffentlicht [1].

Die Forschungsarbeit von S. Golombok et al. [2, 3] bezieht sich auf die stattfindende Evolution der Mitglieder der «neuen» Familien: mit Keimzellenspende (Sperma oder Ovum), mit Leihmutter (durch Schwangerschaft nur oder auch genetisch), gleichgeschlechtliche Eltern, bewusst alleinerziehende Mütter (nicht scheidungsbedingt oder durch abwesenden Partner). Ihre Ergebnisse haben bestimmte Vorstellungen/Befürchtungen zu diesen Familien und zur Entwicklung der Kinder herausgefordert. Sie zeigen, dass die strukturellen Parameter (Anzahl der Personen, Geschlecht, sexuelle Orientierung, genetische Verbindungen oder nicht) für das Wohlergehen der Kinder weniger wichtig sind als die Qualität der familieninternen Beziehungen [3]. «IVF-Familien geht es gut.» Ihre Kinder gedeihen so gut oder so schlecht wie die Kinder aus klassischen Familienstrukturen. Besonderheit: In den Familien mit zwei Müttern sind die "co-mothers" stärker involviert in Bezug auf die Kinder als die Väter. Mehr als 90\% der in homosexuellen Elternhäusern lebenden Kinder sind heterosexuell eingestellt. Eine wesentliche Frage ist die nach der Geheimhaltung (ob künstliche Befruchtung, Keimzellenspende oder Leihmutterschaft). Studien bestätigen, dass Kinder, die informiert wurden (vor allem, wenn dies früh, zwischen dem 4. und dem 7. Lebensjahr erfolgte), besser damit zurechtkommen, als wenn sie unwissend gelassen werden. Bestätigt wird auch, dass die Kinder ganz generell spüren, dass ihnen etwas verborgen wird. "Geheimnisse schaffen Abgrenzungen", sagt Golombok. Dabei sollen die Schwierigkeiten, die IVF-Eltern haben mögen, wenn sie von der medizinisch unterstützten Schwangerschaft sprechen, nicht unter den Tisch gekehrt werden. Aber häufig sprechen Eltern, die ihrem Kind nichts gesagt haben, mit Dritten im Umfeld!!

«IVF-Familien geht es gut.»

Entwicklung in den USA: Das Donor Sibling Registry, ein Register, in dem aus einer Keimzellenspende geborene Kinder ihre Halbbrüder und -schwestern suchen können (bisheriges Maximum: 55 Halbgeschwister, gelegentlich wurden in der Boulevardpresse jedoch höhere Zahlen genannt!). In besagtem Register sind 55000 Mitglieder gelistet, und es kam bislang zu 15000 Kontakten von Betroffenen. Diese Kinder zeigen übrigens (im Jugendlichen- oder Erwachsenenalter) eher Interesse daran, Kontakt mit ihren "half-siblings" aufzunehmen, als mit den Keimzellenspendern. Die Dozentin sprach auch von den Gefühlen und (häufigen!) Beziehungen des Kindes zur Leihmutter.

Abschliessend gilt es auch eine sozietale Dimension zu bedenken. Gelegentlich sind Kinder aus andersartigen Familienstrukturen Gegenstand von unangenehmen Fragen - beispielsweise in der Schule. Während rassistische Äusserungen vom Lehrkörper sofort geahndet werden, ist der Umgang mit Bemerkungen zu familiären Umständen eher zögerlich und weniger strikt. Da bleibt überall noch Handlungsbedarf! 